$$
\text { "tmcs-zeitler" — 2010/11/14 — 22:57 — page } 303 \text { — \#1 }
$$

\title{
Improper integrals, an excursion to classical calculus
}

Herbert Zeitler

Dedicated to my friend Prof. Dr. János Fehér (Pécs)

Abstract. The aim of this paper is to show in what way we investigated improper integrals in the classroom. It was very important to calculate explicitly a lot of examples. Doing so, a criterion was developed. With step curves and infinite series we had a quick look at the mathematical background. The shot in the universe showed that improper integrals are needed in physics.

Key words and phrases: improper integrals, step curves, infinite series, the law of gravity, work and energy, escape velocity.

ZDM Subject Classification: I150.

\section{Some basic facts (without any proof)}

If a region is bounded by a curve $y=f(x)$, the $x$-axis and the straight lines $x=a$ and $x=b$, then the area of this region is $F=\int_{a}^{b} f(x) d x$.

Further we assume, that $f(x)$ is continuous in the interval $[a, b]$ and not negative (Figure 1(a)).

If our region is revolved around the $x$-axis we obtain a special revolution solid (Figure 1(b)). The volume is given by $V=\pi \int_{a}^{b} f^{2}(x) d x$.

Now we calculate the volume of special revolution solids. The function $f(x)$ shall have the following qualities: Continuous in the interval $[1, \infty]$, nonnegative, $f(1)=1$ and monotone decreasing with $\lim _{x \rightarrow \infty} f(x)=0$. Functions of this kind are called special functions and the corresponding curves special curves.

In the following we realize a very important didactical principle: "One must really do it". With this, we have a lot of stimulating activities.

Copyright (C) 2010 by University of Debrecen 


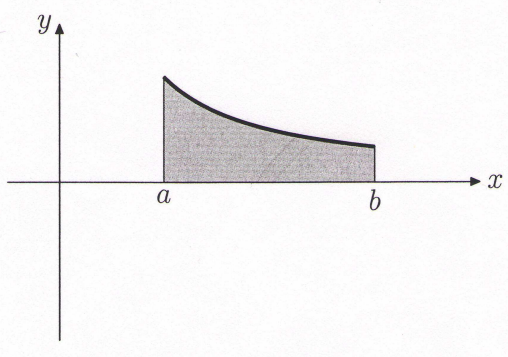

(a) Area of a region

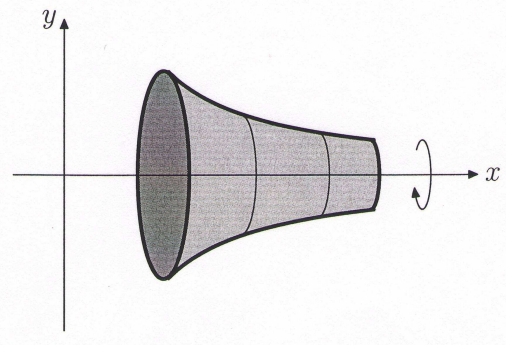

(b) Volume of a revolution solid

Figure 1

\section{Function $f(x)=\frac{1}{x}$}

\subsection{The curve}

The complete curve is symmetric to the origin, running through $(1,1)$. We have an equilateral hyperbola and a pole at the origin. We choose the branch with $x \in[1, \infty]$. Then the curve is monotone decreasing with $\lim _{x \rightarrow \infty} f(x)=0$ and $f(x)>0$. We are working with a special curve (Figure 2).
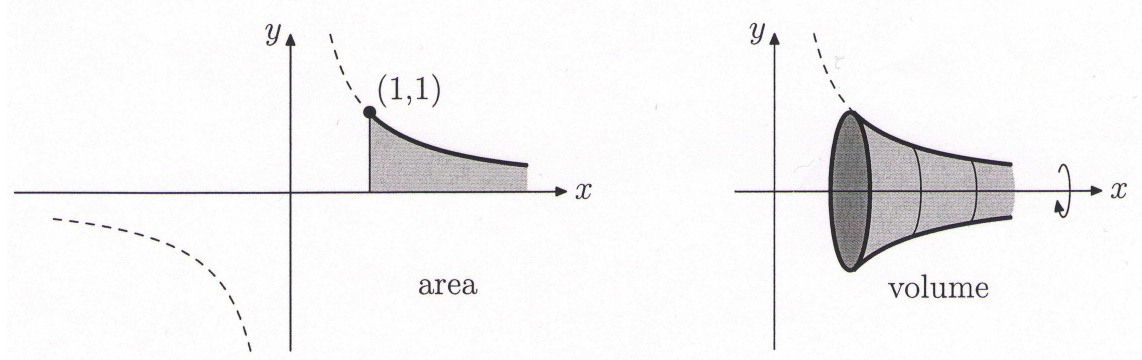

Figure 2. $f(x)=\frac{1}{x}$, area, volume

\subsection{Area}

Due to Chapter 1 the grey region in Figure 2 has the area

$$
F=\int_{1}^{\infty} \frac{1}{x} d x=[\ln x]_{1}^{\infty}=\ln \infty-\ln 1=\infty .
$$




$$
\text { "tmcs-zeitler" — 2010/11/14 — 22:57 — page 305 — \#3 }
$$

\subsection{Volume}

The volume of the corresponding revolution solid is

$$
V=\pi \int_{1}^{\infty} \frac{1}{x^{2}} d x=\pi\left[-\frac{1}{x}\right]_{1}^{\infty}=\pi\left[-\frac{1}{\infty}+1\right]=\pi .
$$

Finally we obtain $F=\infty, V=\pi<\infty$.

REMARK. It is not correct to write $\ln \infty=\infty, \frac{1}{\infty}=0$, because $\infty$ is not at all a number to calculate with. We should write $\lim _{x \rightarrow \infty} \ln x=\infty, \lim _{x \rightarrow \infty} \frac{1}{x}=0$. In respect of a short presentation we keep the wrong spelling and use $\infty$ as number with $\frac{a}{\infty}=0, a \cdot \infty=\infty, a+\infty=\infty, \frac{a}{0}=\infty$ if $a>0$.

\subsection{A problem}

At this point our intuitive ability does not work. We have a revolution solid with finite volume $\pi$. Any plane through the $x$-axis cuts this solid in a region with infinite area. In what way can this work? We can't imagine the situation.

Evangelista Torricelli (1608-1647) already thought about this strange phenomenon. Finally he doubted in the correctness of all mathematics.

We like to mention that Torricelli was a pupil of Galileo Galilei (1564-1642). As Galilei became blind Torricelli read him mathematics and did his correspondence. Torricelli was the first to construct and to use a thermometer. We further know from school that he discovered the famous "Torricelli-vacuum".

\section{A criterion}

Let $f(x)$ be a special function (Chapter 1 ).

(a) If for all $x \in[1, \infty]$ we have $f(x) \geq \frac{1}{x}$ then $\int_{1}^{\infty} f(x) d x=\infty$.

(b) If for all $x \in[1, \infty]$ we have $f(x) \leq \frac{1}{x^{2}}$ then $\int_{1}^{\infty} f(x) d x<\infty$.

The proof follows immediately by comparing $f(x)$ with $\frac{1}{x}$ or $f(x)$ with $\frac{1}{x^{2}}$.

The region $\frac{1}{x^{2}}<f(x)<\frac{1}{x}$ remains open. In this case the criterion does not say anything about convergence or divergence of $\int_{1}^{\infty} f(x) d x$. So our criterion is a very weak one. In spite of this fact the pupils were proud that they had discovered a criterion. Figure 3 illustrates the situation. 


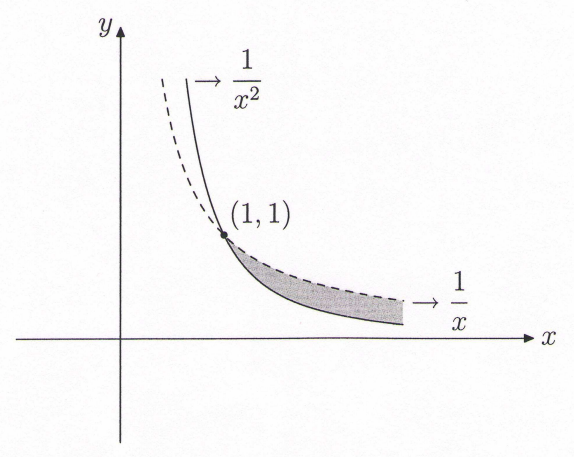

Figure 3. A weak criterion

\section{Function $f(x)=\frac{1}{x^{2}}$}

The complete curve is symmetric to the $y$-axis, running through $(1,1)$, pole at the origin, $f(x)>0$. Now we choose one branch with $x \in[1, \infty]$. Then the curve is monotone decreasing with $\lim _{x \rightarrow \infty} f(x)=0$. We are working with a special curve (Figure 4).
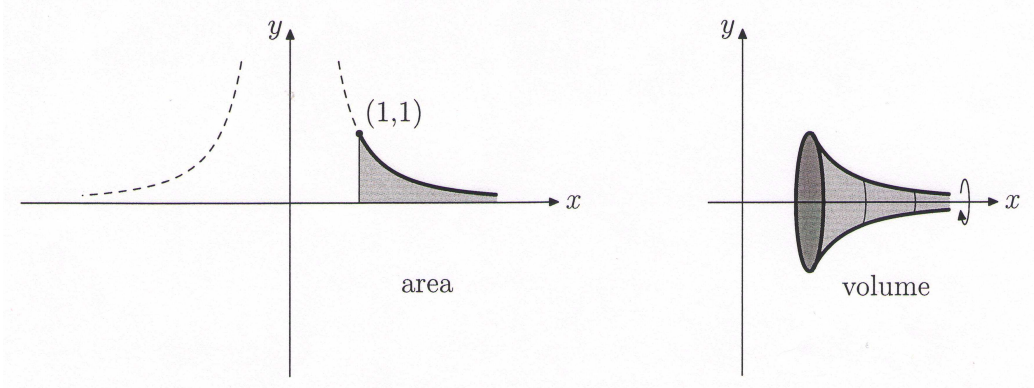

Figure 4. $f(x)=\frac{1}{x^{2}}$, area, volume

\subsection{Area}

$$
F=\int_{1}^{\infty} \frac{1}{x^{2}} d x=\left[-\frac{1}{x}\right]_{1}^{\infty}=-\frac{1}{\infty}+1=1
$$




\subsection{Volume}

$$
V=\pi \int_{1}^{\infty} \frac{1}{x^{4}} d x=\pi\left[-\frac{1}{3 x^{3}}\right]_{1}^{\infty}=\pi\left[-\frac{1}{3 \infty^{3}}+\frac{1}{3}\right]=\pi \frac{1}{3}
$$

Both results were to expect with our criterion:

$$
\frac{1}{x^{2}}=\frac{1}{x^{2}} \Longrightarrow F<\infty, \quad \frac{1}{x^{4}}<\frac{1}{x^{2}} \Longrightarrow V<\infty .
$$

\subsection{An obvious extension}

$$
f(x)=\frac{1}{x^{n}}, \quad n \in \mathbb{N}, n>1
$$

Area

$$
\begin{aligned}
F & =\int_{1}^{\infty} \frac{1}{x^{n}} d x=\left[\frac{x^{-n+1}}{-n+1}\right]_{1}^{\infty}=\left[-\frac{1}{(n-1) x^{n-1}}\right]_{1}^{\infty} \\
& =-\frac{1}{(n-1) \infty}+\frac{1}{n-1}=\frac{1}{n-1}
\end{aligned}
$$

Volume

$$
\begin{aligned}
V & =\pi \int_{1}^{\infty} \frac{1}{x^{2 n}} d x=\pi\left[\frac{x^{-2 n+1}}{-2 n+1}\right]_{1}^{\infty}=\pi\left[-\frac{1}{(2 n-1) x^{2 n-1}}\right]_{1}^{\infty} \\
& =\pi\left[-\frac{1}{\infty}+\frac{1}{2 n-1}\right]=\pi \frac{1}{(2 n-1)}
\end{aligned}
$$

As expected we now have $F<\infty$ and $V<\infty$.

\section{Function $f(x)=\frac{1}{\sqrt{x}}$}

\subsection{The curve}

The curve is running through $(1,1)$, monotone decreasing with $\lim _{x \rightarrow \infty} f(x)$ $=0$, domain $x \in[1, \infty]$, pole at the origin. We are working with a special curve.

\subsection{Area}

$$
F=\int_{1}^{\infty} x^{-\frac{1}{2}} d x=\left[2 x^{\frac{1}{2}}\right]_{1}^{\infty}=2 \infty-2=\infty
$$




\subsection{Volume}

$$
V=\pi \int_{1}^{\infty} x^{-1} d x=\pi[\ln x]_{1}^{\infty}=\pi[\ln \infty-\ln 1]=\infty .
$$

In this case we obtain $F=\infty$ and $V=\infty$.

With criterion:

$$
\frac{1}{\sqrt{x}}>\frac{1}{x} \Longrightarrow F=\infty, \quad \frac{1}{x}=\frac{1}{x} \Longrightarrow V=\infty .
$$

\section{Function $f(x)=\frac{2}{1+x^{2}}$ versiera}

The versiera was investigated for the first time, by Maria Agnesi (1718-1799), the angle of the poor people. Unfortunately versiera was translated in a wrong way as "aversiera" - this means "wife of the devil". Therefore sometimes the versiera is denoted as "witch of Agnesi".

\subsection{The curve}

The curve is running through $(1,1), f(x)>0$, maximum at $(0,2)$, in the domain $x \in[1, \infty]$ the curve is monotone decreasing with $\lim _{x \rightarrow \infty} f(x)=0$ and $f(x)>0$ (Figure 5). We are working with a special curve.
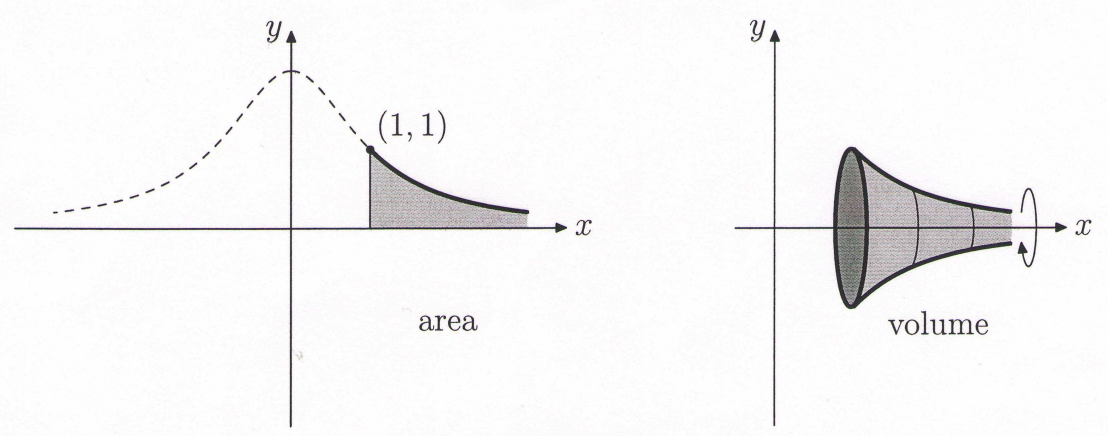

Figure 5. $f(x)=\frac{2}{1+x^{2}}$, area, volume 


$$
\text { "tmcs-zeitler" — 2010/11/14 — 22:57 — page } 309 \text { — \#7 }
$$

\subsection{Area}

$$
F=\int_{1}^{\infty} \frac{2}{1+x^{2}} d x=2[\arctan x]_{1}^{\infty}=2[\arctan \infty-\arctan 1]=2\left[\frac{\pi}{2}-\frac{\pi}{4}\right]=\frac{1}{2} \pi
$$

\subsection{Volume}

$$
V=\pi \int_{1}^{\infty} \frac{4}{\left(1+x^{2}\right)^{2}} d x
$$

In a collection of formulas we find the indefinite integral

$$
\int \frac{1}{\left(1+x^{2}\right)^{2}} d x=\frac{1}{2}\left[\frac{x}{1+x^{2}}+\arctan x\right]+C .
$$

To prove this formula we only calculate the derivative with the usual techniques. With this we obtain

$$
\begin{aligned}
V & =\pi \int_{1}^{\infty} \frac{4}{\left(1+x^{2}\right)^{2}} d x=\frac{4 \pi}{2}\left[\frac{x}{1+x^{2}}+\arctan x\right]_{1}^{\infty} \\
& =2 \pi\left[\frac{\infty}{\left(1+\infty^{2}\right)^{2}}+\arctan \infty-\frac{1}{2}-\arctan 1\right] \\
& =2 \pi\left[\frac{\pi}{2}-\frac{1}{2}-\frac{\pi}{4}\right]=\frac{1}{2} \pi[\pi-2] .
\end{aligned}
$$

It turns out that $F<\infty$ and $V<\infty$.

\subsection{And our comparison criterion?}

It turns out that $\frac{2}{1+x^{2}}<\frac{1}{x}$ and $\frac{2}{1+x^{2}}>\frac{1}{x^{2}}$. In this case our criterion does not work. With this we know that the inverse of criterion 3(b) can't hold. Further we find $\frac{4}{\left(1+x^{2}\right)^{2}}<\frac{1}{x^{2}}$ and then $V<\infty$.

\section{Finite or infinite - that's the question}

\subsection{The possibilities}

In the following table we give all combinations for $F$ and $V$. Further we name corresponding examples. 
One case remains open.

\begin{tabular}{c|c|c}
$F$ & $V$ & examples \\
\hline$\infty$ & $\infty$ & $\frac{1}{\sqrt{x}}$ \\
$\infty$ & $<\infty$ & $\frac{1}{x}$ \\
$<\infty$ & $\infty$ & $?$ \\
$<\infty$ & $<\infty$ & $\frac{1}{x^{2}}$, versiera
\end{tabular}

\subsection{The question mark}

The case $F<\infty$, and $V=\infty$ never occurs.

Proof. Because $f(x)$ is a special function $(f(1)=1$, nonnegative, decreasing with $\left.\lim _{x \rightarrow \infty} f(x)=0\right)$ there exists a number $b>1$ with $f(x)<1$ if $x>b$. Therefore $f^{2}(x)<f(x)$ and $f^{2}(x)-f(x)<0$. Thus we obtain

$$
\begin{aligned}
V & =\pi \int_{1}^{\infty} f^{2}(x) d x \\
& =\pi \int_{1}^{b} f^{2}(x) d x+\pi \int_{b}^{\infty} f^{2}(x) d x<\pi \int_{1}^{b} f^{2}(x) d x+\pi \int_{b}^{\infty} f(x) d x \\
& =\pi \int_{1}^{b} f^{2}(x) d x+F-\pi \int_{1}^{b} f(x) d x=\pi \int_{1}^{b}\left(f^{2}(x)-f(x)\right) d x+F<F .
\end{aligned}
$$

With $F<\infty$ finally we obtain $V<\infty$. The case $F<\infty$ and $V=\infty$ never occurs.

\section{Another proof of our criterion}

8.1. What we did and what we like to do

Our criterion in Section 3 is based on two definite integrals $\int_{1}^{\infty} \frac{1}{x} d x, \int_{1}^{\infty} \frac{1}{x^{2}} d x$. These integrals were found using the well-known integral techniques. Now we give another proof without this machinery. Doing so, we must dive in the background, in the mysteries of integral theory. There we need the concept of step-curves 


$$
\text { "tmcs-zeitler" — 2010/11/14 — 22:57 — page } 311 \text { — \#9 }
$$

(Figures 6, 7) and two infinite series

$$
\begin{aligned}
& 1+\frac{1}{2}+\frac{1}{3}+\cdots=\sum_{1}^{\infty} \frac{1}{n}=\infty, \quad \text { harmonic series } \\
& 1+\frac{1}{2^{2}}+\frac{1}{3^{2}}+\cdots=\sum_{1}^{\infty} \frac{1}{n^{2}}=\frac{1}{6} \pi^{2} .
\end{aligned}
$$

\subsection{A first approximation}

The function $f(x)$ is a special one and therefore monotone decreasing with $\lim _{x \rightarrow \infty} f(x)=0$.

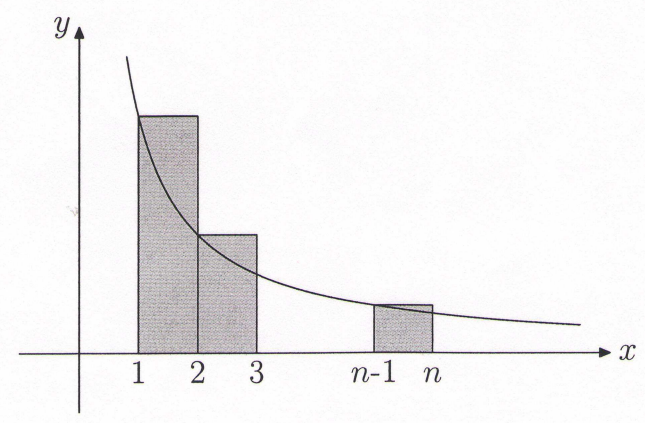

Figure 6. A first approximation

Now we take from Figure 6 the following approximation sum

$$
\begin{aligned}
\int_{1}^{n} f(x) d x & =\int_{1}^{2} f(x) d x+\int_{2}^{3} f(x) d x+\cdots+\int_{n-1}^{n} f(x) d x \leq \\
& \leq \Delta x f(1)+\Delta x f(2)+\cdots+\Delta x f(n-1) \quad(\text { and with } \Delta x=1) \\
& =f(1)+f(2)+\cdots+f(n-1) .
\end{aligned}
$$

In the case $f(x)=\frac{1}{x^{2}}$ we obtain with our infinite series

$$
\int_{1}^{\infty} \frac{1}{x^{2}} d x \leq \sum_{1}^{\infty} \frac{1}{n^{2}}=\frac{1}{6} \pi^{2} .
$$

The sequence $\int_{1}^{n} \frac{1}{x^{2}} d x, n \in \mathbb{N}$ is increasing in a monotone way and bounded. Therefore the limes $\int_{1}^{\infty} \frac{1}{x^{2}} d x$ really exists. 


\subsection{A second approximation}

$f(x)$ is again a special function.

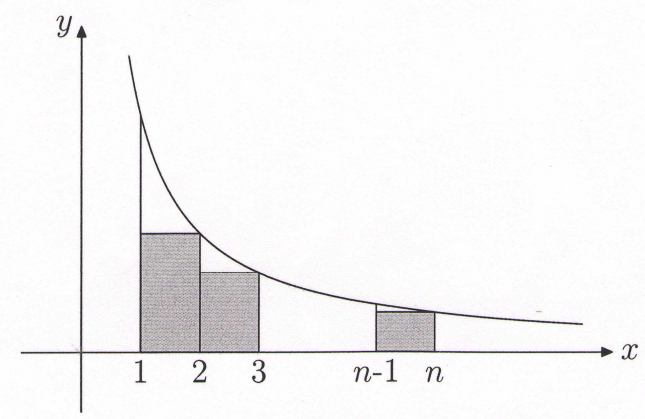

Figure \%. A second approximation

We take from Figure 7 the following approximation sum

$$
\begin{aligned}
\int_{1}^{n} f(x) d x & =\int_{1}^{2} f(x) d x+\int_{2}^{3} f(x) d x+\cdots+\int_{n-1}^{n} f(x) d x \geq \\
& \geq \Delta x f(2)+\Delta x f(3)+\cdots+\Delta x f(n) \quad(\text { and with } \Delta x=1) \\
& =f(2)+f(3)+\cdots+f(n) .
\end{aligned}
$$

In the case $f(x)=\frac{1}{x}$ this yields

$$
\int_{1}^{\infty} \frac{1}{x} d x \geq \sum_{2}^{\infty} \frac{1}{n}=\infty .
$$

The sequence $\int_{1}^{n} \frac{1}{x} d x, n \in \mathbb{N}$ is running to $\infty$. With $8.2,8.3$ we have again $\int_{1}^{\infty} \frac{1}{x^{2}} d x<\infty$ and $\int_{1}^{\infty} \frac{1}{x} d x=\infty$.

\section{Other fields for working with students}

\subsection{Improper integrals}

In this paper we have investigated integrals of the form $\int_{1}^{\infty} f(x) d x$, where $f(x)$ was a special function. The domain was infinite. 


$$
\text { "tmcs-zeitler" — 2010/11/14 — 22:57 — page 313 — \#11 }
$$

Definition. All integrals over a function with infinite domain are called improper. (The domain may be infinite to both sides.)

Now we consider a function $f(x)$ continuous over a finite domain $[a, b]$. At $x=a$ shall be a pole with a vertical asymptote. So the range is infinite.

$\int_{a}^{b} f(x) d x=\lim _{\varepsilon \rightarrow 0} \int_{a+\varepsilon}^{b} f(x) d x$. Integrals of this kind are also called improper.

Activities. $f(x)=\sqrt{\frac{x^{3}}{1-x}}($ cissoid), range infinite, domain $[0,1]$. To show: $F=\int_{0}^{1} \sqrt{\frac{x^{3}}{1-x}} d x=\frac{3}{8} \pi$. Calculate the volume of the corresponding revolution solid (rotating around the $x$-axis). Look for other examples of improper integrals!

\subsection{Other transitions}

In this paper we investigated the transition from dimension 2 to dimension 3 , from area to volume. It is only natural that other transition also exist, for instance from 1 to 2 , from length to area.

The so-called Koch-curve (show flake) is a very nice example for this kind of transition (Figure 8).
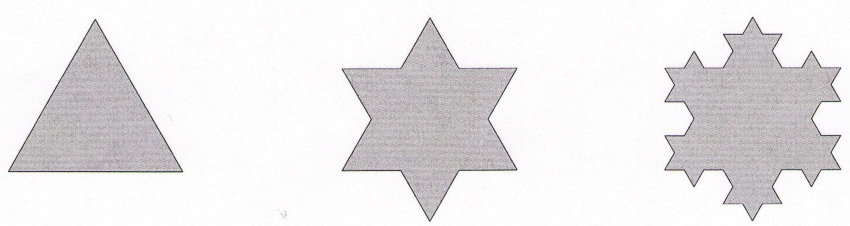

Figure 8. Koch-curve, shown flake

If one side of the starting equilateral triangle has length a, then the total length of the Koch-curve is infinite, but the area of the enclosed point set is $F=\frac{2}{5} \sqrt{3} a^{2}$. We have again a problem with our intuitive ability.

ACtivities. Look for other transitions and calculate!

Doing so, the fantasy has no limits. 


$$
\text { "tmcs-zeitler" — 2010/11/14 — 22:57 — page 314 — \#12 }
$$

\section{Shot in the universe}

Now we like to show that improper integrals are really needed in science. Only one example from physics is investigated here. Unifortunately a lot of fundamentals from physics are needed.

\subsection{The law of gravity}

We consider an object with mass $m$ (for instance a satellit) in the surrounding of the earth with mass $M$, and $m \ll M$. The radius of the earth is $R$, the distance between the object and the center of the earth is $x$ (Figure 9 ).

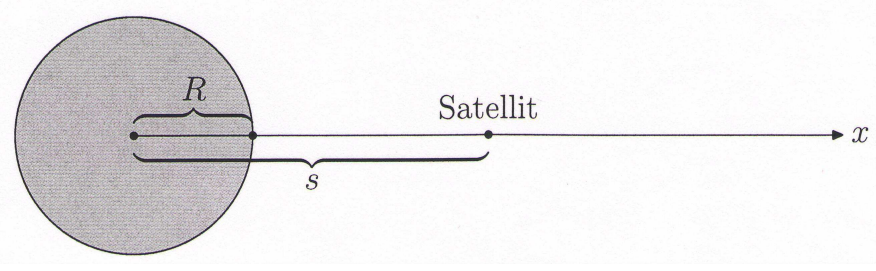

Figure 9. Shot in the universe

Then the force of gravity between the two masses is

$$
F=f \frac{m M}{x^{2}}
$$

$f$ is the so-called gravitational constant. With difficult experiments scientists found $f \approx 6.69 \cdot 10^{-11} \frac{\mathrm{m}^{3}}{\mathrm{~kg} \mathrm{sec}^{2}}$.

\subsection{Work and energy}

To transport our object at a point with distance $s$ from the earth-center we need the work

$$
E_{s}=\int_{R}^{s} \frac{f m M}{x^{2}} d x .
$$

If the object shall fly in the universe and never come back we need the energy

$$
E_{\infty}=\int_{R}^{\infty} \frac{f m M}{x^{2}} d x=f m M\left[-\frac{1}{x}\right]_{R}^{\infty}=\frac{f m M}{R}
$$




$$
\text { "tmcs-zeitler" — 2010/11/14 — 22:57 — page 315 — \#13 }
$$

\subsection{Escape velocity}

If our object shall fly in the universe, we must give it the kinetic energy $E_{\infty}$. Let $v_{0}$ be the escape velocity, then we have

$$
\frac{1}{2} m v_{0}^{2}=\frac{f m M}{R} \Longrightarrow v_{0}=\sqrt{\frac{2 M f}{R}} .
$$

For further calculation we need some more information.

In the starting point on earth we have the gravitational force $F$ between the two solids. This is just the weight of our object. This means

$$
m g=\frac{f m M}{R^{2}} \Longrightarrow f M=g R^{2} .
$$

The constant $g$ is the acceleration due to the gravitational attraction of the earth. Experiments yield $g \approx 9.81 \frac{\mathrm{m}}{\mathrm{sec}^{2}}$.

We substitute the last result in the former $v_{0}=\sqrt{\frac{2 f M}{R}}=\sqrt{2 g R}$. With $R \approx 6.37 \cdot 10^{6} \mathrm{~m}$ we obtain the escape velocity

$$
v_{0} \approx 11.18 \frac{\mathrm{km}}{\mathrm{sec}} .
$$

REMARK. The escape velocity is $v_{0}$ if we have a direct shot without some rocket propulsion.

There remain questions. One example: What happens, if the object is not fired in the prolongation of an earth radius.

Activities. Calculate the mass $M$ of the earth, using the values of $g, f, R$.

\section{A medly with various ideas}

\subsection{Dreams about didactics}

My idea in mathematical education for many, many years was to stimulate pupils and school teachers. They should do creative mathematics for their own. To find a tiny theorem is much more important (and satisfactory too) as to study scholarly books. Students and teachers should become little researchers. Then doing mathematics is a real pleasure.

Therefore I require from ministeries oasis in classrooms to do nice mathematics. I preach the didactics of joy.

The investigations around the improper integrals - as treated in this paper and with pupils - is a very good way to realize my didactical dreams. 


$$
\text { "tmcs-zeitler" — 2010/11/14 — 22:57 — page 316 — \#14 }
$$

\subsection{From the lumber room}

At the end of the 20th century our pupils in gymnasium had to calculate the volume of revolutions solids - and other things like this. In connection with our present paper we give two exercises from school books of this time.

EXERCISE 1. A parabola is rotating around its axis. This yields a paraboloid.

To Prove. The volume of a revolution paraboloid over a circle with radius $r$ is half of the volume of a cylinder over this circle enclosing the paraboloid. The paraboloid is inscribed the cylinder.

REMARK. Over a circle of radius $r$ a cylinder with height $r$ is constructed. In this cylinder are inscribed a semisphere, our paraboloid from Exercise 1 and a cone. Figure 10 shows the situation.
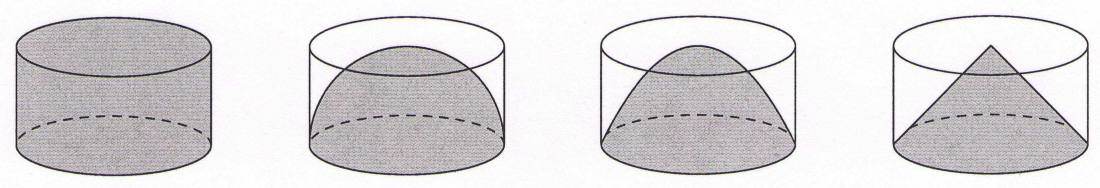

Figure 10. Four revolution solids

The volume of these solids may be calculated. Here we give the results:

$$
V_{1}=\pi r^{3}, \quad V_{2}=\frac{2}{3} \pi r^{3}, \quad V_{3}=\frac{1}{2} \pi r^{3}, \quad V_{4}=\frac{1}{3} \pi r^{3} .
$$

So we obtain the following volume-ratios:

$$
V_{1}: V_{2}: V_{3}: V_{4}=6: 4: 3: 2 .
$$

Now we tell a story concerning Archimedes (287-212 B.C.). On the tomb of Archimedes a stone was erected with the pictures of a cylinder and a inscribed semisphere (these are exactly the first two cases in Figure 10). Further on the tomb-stone the ratio $3: 2$ was engraved, to remember one of the greatest discovery of Archimedes.

Archimedes himself wanted this very special monument when he was still alive. 


$$
\text { "tmcs-zeitler" — 2010/11/14 — 22:57 — page } 317 \text { — \#15 }
$$

EXERCISE 2. Given a straight line with equation $x-2 a=0$ and a point $P(a, b)$. We are looking for a parabola through $P$, opened to the right, $x$-axis is parabola axis. The segment formed by the line and the parabola is rotating around the $x$-axis. In this way a revolution solid is born. The volume of this solid shall be minimal.

What is the equation of the parabola and what is the minimal volume?

You - the reader - should try to solve these old fashioned exercises.

Actually it's a pity that this field of mathematics - revolution solids - is disappeared in the schools. Sorry to say!

\subsection{The death of school geometry}

Some provocative final remarks.

Teaching in our gymnasium has totally changed in the last years here in Germany. Fundamental calculation-techniques are missing. Geometric visualizations and geometry as a whole is shrinking - step by step - towards zero. But I claim that without geometry our school mathematics is a pale, naked skeleton without flesh and blood.

Is it still possible to stop this catastrophal process or to turn it back? I believe, I fear: NO.

\section{References}

[1] H. Dörrie, Unendliche Reihen, München, 1951.

[2] E. Hille, Analysis volume I, New York, 1964.

[3] H. Schupp and H. Dabrock, Höhere Kurven, Mannheim, 1995.

[4] H. Zeitler and D. Pagon, Fraktale Geometrie, Braunschweig, 2000.

[5] Various school books.

HERBERT ZEITLER

BAYREUTH

GERMANY

(Received May, 2010) 